\title{
De ciudadanías insurgentes a planificadores, urbanos. Organización social en la urbanización del campamento Manuel Bustos de Viña del Mar ${ }^{1}$
}

\section{From insurgent citizenships to urban planners. Social organization in the urbanization of the Manuel Bustos slum in Viña del Mar}

\author{
Elizabeth Zenteno Torres ${ }^{2} \odot$, Kimberly Sepúlveda Muñoz ${ }^{3} \odot$,

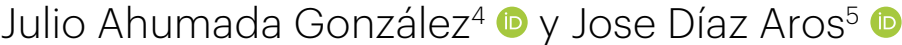

\begin{abstract}
RESUMEN
Después de varias décadas de instalado el modelo de política pública en Chile, se hace evidente que la promesa del fin de campamentos fue incumplida. Los asentamientos informales han persistido y continuado en expansión desde los años ' 90 . A través de un trabajo etnográfico, se expone el proceso de formación y urbanización del campamento más grande de Chile: Manuel Bustos. La trayectoria de colaboración para producir su propio hábitat, la formación y empoderamiento de dirigencias sociales son la base de la organización comunitaria del campamento, lo cual ha permitido quebrar la lógica topdown de las políticas de vivienda, poniendo en práctica un modelo de planificación participativa. La participación social permite posicionar sus demandas en la agenda pública y concretar la urbanización. Este proceso responde no sólo al requerimiento de derecho a la vivienda, sino al derecho a la ciudad; transformando ciudadanías insurgentes, formadas en el campamento, en competentes planificadores urbanos.

Palabras clave: organización comunitaria, vivienda, asentamientos informales, urbanización, campamentos.
\end{abstract}

Investigación financiada por el Proyecto Fondecyt Postdoctoral № 3190271 “Apropiación y resignificación como disputa del espacio urbano. Prácticas y sentidos de la ciudad en migrantes latinoamericanos" y por el Proyecto de la Dirección General de Investigación de la Universidad de Playa Ancha № № 17-1718: "Campamentos en Viña del Mar y Valparaíso, una mirada desde los espacios de relegación".

Instituto de Estudios Urbanos y Territoriales, Pontificia Universidad Católica de Chile / Observatorio de Participación Social y Territorio, Facultad de Ciencias Sociales, Universidad de Playa Ancha. Correo electrónico: elizabeth.zenteno@upla.cl

Observatorio de Participación Social y Territorio, Facultad de Ciencias Sociales, Universidad de Playa Ancha. Correo electrónico: kimberly.sepulveda.munoz@gmail.com

Observatorio de Participación Social y Territorio, Facultad de Ciencias Sociales, Universidad de Playa Ancha. Correo electrónico: julio.ahumada.g@gmail.com

Observatorio de Participación Social y Territorio, Facultad de Ciencias Sociales, Universidad de Playa Ancha. Correo electrónico: diazaros.joseignacio@gmail.com 


\begin{abstract}
Several decades have passed since the installation of the Chilean Model of Public Policy, but the promise of 'the end of slums' is still yet to be fulfilled. Despite the government's claimed intentions, informal settlements have persisted and have even expanded since the '90s. A recent ethnographic study has exposed the formation and urbanization of Chile's largest slum: Manuel Bustos, Viña del Mar. The community is founded on principles of collaboration and the empowerment of social leadership, both of which have enabled a breakaway from the top-down logic of national housing policies. Instead, the Manuel Bustos community has implemented an independent model of participatory planning that produces and maintains habitat formation. Broad social participation has allowed the community to position their demands on the public agenda, resulting in the specification of urbanization. This process is a response not only to the requirement of the right to adequate housing, but also to the right to the adequate services that cities provide. In their own right, residents of Manuel Bustos have transformed their status from insurgent citizenships formed in the slum, into competent urban planners of an independently founded city.
\end{abstract}

Keywords: community organization, housing, informal settlement, urbanization, slum.

El nacimiento de los campamentos en Chile, al igual que en el resto de América Latina, estuvo determinado por la movilización de un gran componente migratorio proveniente de las áreas rurales y que, en la contingencia, se estableció en espontáneas y precarias moradas. A estas moradas se les conoció, entonces, como callampas por la rapidez con la que allí surgían. A mediados de los años 60, la conformación de campamentos estuvo estrechamente ligada a organizaciones sociales de personas 'sin casa' que ocupaban o 'se tomaban' el terreno (Zenteno, 2015). La toma de sitios fue una modalidad de reivindicación política cuya práctica, posteriormente, se extendió por diversas zonas del país como "estrategia que iría ganando en prestigio y desarrollo en los años siguientes: si el Estado no atendía las demandas por la vivienda de los pobladores, ellos mismos, organizadamente, podían tomar sitios y levantar sus propias poblaciones" (Garcés, 2015:35). Las ocupaciones masivas de terreno, propias de este periodo, fueron altamente politizadas y construyeron diversos tipos de alianza, ya sea con la Iglesia, partidos políticos o con los sectores más progresistas de las clases medias (Angelcos \& Pérez, 2017).

Con posterioridad a la ocupación del terreno, en general, se procedió a una serie de movilizaciones sociales para obtener los servicios de agua, luz, alcantarillado y pavimentación. Las movilizaciones orientadas a la obtención de vivienda y equipamiento urbano fueron especialmente activas entre 1970 y 1972 (Garcés, 2015), en el periodo de la presidencia de Salvador Allende. Durante la Unidad Popular, muchas de estas nuevas tomas o campamentos se arraigaron en el territorio y, luego, comenzaron a ser denominadas como poblaciones (Cortés, 2014). Con la violenta represión a los movimientos sociales durante la dictadura, las tomas de terreno disminuyen significativamente. Por lo demás, se implementa un modelo neoliberal que implica un cambio en la forma de acceder a la vivienda: deja de ser un derecho colectivo para ser individualizados en cada una de las familias que, mediante mecanismos de ahorro individual, pueden acceder a programas de subsidios de vivienda (Özler, 2012). Esta política de vivienda crea en el discurso público la ilusión del 'fin de los campamentos' a través de la construcción de vivienda a gran escala, fenómeno que redundó en un éxodo masivo desde campamentos a viviendas sociales (Skewes, 2005).

Algunos estudios (Ducci, 1997) señalan que estas características del modelo chileno de vivienda social, centrado en el ahorro individual, ha sido particularmente eficiente en reducir la 
demanda habitacional, que en la práctica ha implicado la construcción masiva de vivienda nueva, con materiales de baja calidad, sin servicios y alejada de la ciudad lo que ha generado otros problemas asociados (Rodríguez \& Sugranyes, 2004).

Simultáneamente a la implementación de este modelo de política pública, en las periferias de algunas ciudades del país fueron germinando silenciosamente algunos campamentos. Desde el año 2007 los asentamientos informales en Chile aumentan progresivamente, pasando de ser 490 campamentos a 802 el año 2019 (MINVU, 2019). La Región de Valparaíso destaca por ser la zona de Chile que cuenta con mayor número de asentamientos informales (181), dentro de la cual Viña del Mar sobresale tanto por el número de campamentos (70), como de hogares residiendo en ellos (6.627). El campamento más grande de Chile, Manuel Bustos, se sitúa en la ciudad de Viña del Mar y desde el año 2017 se encuentra en proceso de urbanización. El objetivo de este artículo es, en primer lugar, analizar la conformación del campamento a la luz de la producción de su propio hábitat, describiendo los procesos de ocupación del espacio, de infraestructura, pero también de construcción de comunidad. En segundo lugar, analizar cómo la dirigencia comunitaria se ha configurado en el proceso de urbanización del campamento y de qué modo la participación ciudadana se ha inmiscuido en la planificación y ejecución de la política pública, develando el rol que ha jugado la dirigencia comunitaria en esta transformación de ciudadanías insurgentes a planificadores urbanos, que demandan más que el derecho a la vivienda, el derecho a la ciudad.

El caso del campamento Manuel Bustos es relevante por diversos motivos que van más allá de la cantidad de residentes que tiene. Ante todo, es relevante porque en él se está aplicando una nueva política de urbanización de asentamientos informales, después de décadas de funcionamiento sistemático de un modelo de política pública de vivienda de remoción de los campamentos (Ducci, 1997). La urbanización del campamento Manuel Bustos constituye un emblema de una nueva política de vivienda participativa y, de paso, señala un reconocimiento a las nefastas consecuencias que ha provocado el modelo precedente (Rodríguez \& Sugranyes, 2004). Lo novedoso de esta política pública es que prácticamente desde hace cuarenta años que en Chile no se ejecuta la urbanización de campamentos. En la política pública reciente ha habido programas de remoción y reasentamiento en la misma comuna (Salcedo, 2010), no así de urbanización del mismo territorio habitado. Asimismo, el proceso de urbanización ha dado pie a una instancia participativa que incluye a las organizaciones que agrupan a los pobladores del territorio en la planificación de soluciones comunitarias -ya no individuales- en el proceso planificación urbana.

La participación comunitaria en la construcción de ciudad ha sido reconocida en el actual movimiento de allegados, entendiéndola como una continuidad del movimiento de pobladores (Angelcos \& Pérez, 2017), propuesta que disputa con la idea del desamparo y desarticulación de la organización social de los barrios (Koppelman, 2016; Özler, 2012). En los campamentos, Brain, Prieto \& Sabatini (2010) exploran en los significados asociados al vivir en estos territorios, en vistas del progresivo aumento desde los años 2000. Más recientemente en el debate académico la noción de campamento ha estado asociada a la llegada de migrantes a Chile (Contreras et al., 2019; López-Morales et al., 2018). Sin embargo, existe un vacío en los estudios que indagan en los campamentos contemporáneos de las ciudades intermedias, lo cual es aún más evidente en la región de Valparaíso, la cual paradójicamente, reúne el mayor número de estas formas de habitar. Específicamente, los campamentos en la ciudad de Viña del Mar parecen haber sido invisibles por décadas no sólo frente al debate público y político sino también para el desarrollo científico. Este artículo viene a suplir este vacío en la producción académica. 
El documento está dividido en cuatro partes. En la primera presentamos los antecedentes teóricos desde los cuales se ha entendido la participación ciudadana en la construcción de ciudad. En segundo lugar, exponemos en mayor detalle el caso de estudio y los métodos utilizados. La tercera sección está dedicada a los resultados, los que están organizados en dos partes: primero se analiza la conformación del campamento Manuel Bustos, tanto a nivel de infraestructura como comunitario; para luego analizar el papel que han tenido las dirigencias en el ejercicio de planificación participativa, logrando transformar las demandas de derecho a la vivienda en derecho a la ciudad. Finalmente, en las reflexiones finales proponemos algunas recomendaciones de política pública.

\section{Participación en la construcción de ciudad: De producción social del hábitat a planificación participativa.}

Muchas ciudades del Sur Global han sido en gran parte construidas por sus propios residentes, quienes construyen sus viviendas y barrios (Caldeira, 2017). Por lo general, esta práctica se produce por parte de los sectores medios-bajos como consecuencia a la alta brecha entre la producción capitalista de vivienda y la demanda de vivienda y hábitat (Di Virgilio \& Rodríguez, 2013). Caldeira (2017) recurre al término urbanización periférica, para referirse a aquel modo de producción del espacio en la cual los propios residentes construyen su vivienda, calles y barrios.

En los últimos años dentro del debate académico ha adquirido importancia la noción de Producción Social del Hábitat, para poder comprender las lógicas de habitabilidad y lucha por el suelo urbano. Dicha noción, enfatiza la interacción multiactoral (Boldrini \& Gómez, 2014; Di Virgilio \& Rodríguez, 2013), que se expresa en la autoproducción social del hábitat y el derecho a la ciudad, en contraposición con las lógicas de producción capitalista de la vivienda y el papel activo del mercado dentro de respectivas temáticas. Esta noción que permite comprender la forma en que sectores populares construyen su espacio buscando solucionar sus problemáticas de vivienda por sus propios medios, incorporando también el entorno social y ambiental en el cual se reside (González, 2012).

Este enfoque resalta la necesidad de concebir a los pobladores como actores sociales que desarrollan mecanismos para el acceso al suelo urbano a través de lógicas colaborativas y comunitarias (Di Virgilio \& Rodríguez, 2013; Arqueros Mejica et al., 2009), y por otro lado, el rechazo a una figura estatal que delega su rol dentro de las temáticas de vivienda al mercado quien construye la imagen de la vivienda como un bien de mercado, eliminando su dimensión social, cultural y humana (Ortiz, 2012; Sugranyes, 2012).

El acceso al suelo producto a la especulación, la falta de recursos para llevar a cabo proyectos colaborativos y la limitación jurídica-legal se constituyen como problemáticas centrales al momento de hablar de PSH. Desde esta misma línea, los pobladores se enfrentan constantemente a la deslegitimación política y a la desvalorización de las herramientas que ellos poseen para construir ciudad. Frecuentemente son considerados como violadores el orden urbanístico, cuyo 'crimen' es no obedecer a la planificación, locus donde se definen las formas permitidas -o prohibidas- de organizar el espacio (Rolnik, 2017). 
Enfoques innovadores de planificación urbana están rescatando la experiencia de producir su propio hábitat de las comunidades, en la urbanización de asentamientos informales. Este nuevo enfoque de política pública está dando espacio para la superación de las relaciones dominador-dominado, intentando una ruptura con los roles instituidos. Si bien los discursos oficiales aluden y reconocen la relevancia de las comunidades en la formulación de políticas públicas, dicho protagonismo no necesariamente se refleja en procesos consistentes ni en instrumentos claros de participación y vinculación ciudadana (Lange, 2017). De hecho, el acceso concreto a la toma de decisiones es el núcleo fundamental de la participación ciudadana (Boldrini \& Gómez, 2014).

Por otro lado, es necesario tener en cuenta que la participación de la sociedad civil en políticas públicas no ha sido una graciosa concesión del Estado, sino una conquista de los movimientos sociales y un resultado del despertar de la sociedad civil que reclama, a través de las organizaciones, un lugar en la toma de decisiones (Sarmiento et al., 2007). Frente a los procesos participativos que se están creando, que abren caminos para que la sociedad civil sea parte de la planificación -formal- de la ciudad.

\section{Caso de estudio y métodos}

En este documento se presentan los resultados de una investigación etnográfica realizada en el campamento Manuel Bustos ubicado en la ciudad de Viña del Mar, en la región de Valparaíso,

Figura N01.

Campamento Manuel Bustos, Viña del Mar.
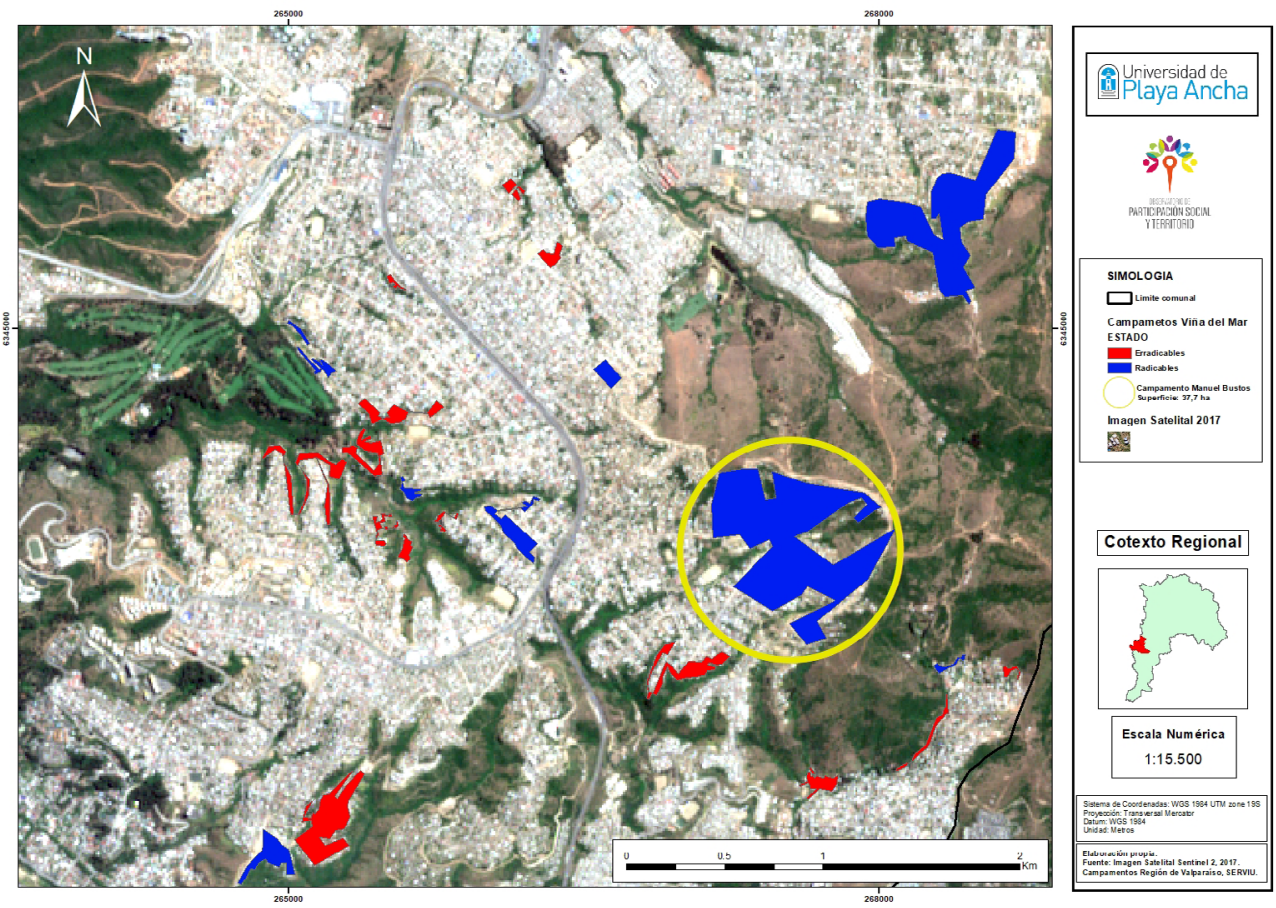

Fuente: Elaboración propia en base a información SERVIU, 2017. Imagen satelital Sentinel 2, 2017 
el cual comenzó en marzo del 2017 y se ha extendido hasta fines de 2019. El método utilizado ha sido el etnográfico, con particular énfasis en los distintos posicionamientos de las investigadoras e investigadores, pues nuestro trabajo integra diversas perspectivas: trabajamos conjuntamente investigadores externos junto a una investigadora nativa, socióloga, residente desde hace más de 15 años del campamento Manuel Bustos. Todo el equipo ha sido parte del trabajo de campo, en distintos momentos y episodios, por ello, haciendo referencia al trabajo de Auyero \& Swistun (2008), podríamos decir que nuestro trabajo es una etnografía cubista.

Manuel Bustos se constituye como el campamento más grande de la ciudad de Viña del Mar, contando con un total de 1.647 familias según los últimos datos entregados por el MINVU (2019). Dentro de la figura $\mathrm{N}^{\circ} 1$, los campamentos marcados son aquellos considerados en el Convenio entre el Gobierno central a través del Ministerio de Vivienda (MINVU) y el Gobierno Regional de Valparaíso ${ }^{6}$. Esta alianza busca ser una respuesta a los campamentos que han surgido en la región, por lo cual identifica a los campamentos, estableciendo una modalidad de regularización, ya sea mediante la radicación o erradicación (relocalización). Los campamentos marcados con azul, -donde Manuel Bustos está marcado con un círculo amarillo-, son aquellos que están contemplados con la alternativa de radicación en el mismo territorio, mientras que los marcados con rojo, son aquellos planificados para reubicación.

Nuestro trabajo etnográfico ha integrado participación en las reuniones de los comités de vivienda, reuniones entre las instituciones y los comités, participación en actividades comunitarias, pero también se integran otras técnicas complementarias al trabajo, como lo fue la realización de 18 entrevistas a representantes de instituciones públicas, incluyendo SERVIU y Municipalidad de Viña del $\operatorname{Mar}^{7}(3)$, dirigentes sociales (8) y residentes de Manuel Bustos (7).

\section{Formar un campamento, conformar comunidad}

Las primeras viviendas que llegan al campamento Manuel Bustos se establecen en torno a los años 90 y son parte de una segunda generación de tomas. La ciudad de Viña del Mar, en el periodo previo a la dictadura ya conocía movimientos de pobladores. No obstante, en esta segunda etapa post-dictatorial de tomas de terrenos, las ocupaciones tienen un componente que las distingue de aquellas ocurridas antes del golpe. En los 90 , las tomas de terrenos son menos politizadas y menos colectivas que en los 60; si se quiere, son estrategias unifamiliares desplegadas por los pobladores ante la carencia de viviendas, ya no, acciones colectivas de reivindicación de derechos sociales. Por ello, es difícil establecer una fecha exacta en la que se inicia el campamento Manuel Bustos, ya que es producto de una ocupación progresiva de tomas, desalojos y nuevamente tomas del terreno. Solo uno de los 23 comités de vivienda ${ }^{8}$ que actualmente conforman

\footnotetext{
El Convenio fue firmado el año 2013 y actualizado el año 2018. Con su renovación el año 2018 se extiende el plazo hasta el 2025 para ejecución y, por lo demás, se incluye dentro del Convenio campamentos que surgieron después del 2013.

Las entrevistas a representantes de las instituciones públicas se realizaron el año 2017, durante el segundo gobierno de Bachelet. Durante los años 2019 y 2020, en el gobierno de Piñera, se intentó en diversas instancias replicar las entrevistas, las cuales no nos fueron concedidas.

8 El comité de vivienda es una organización funcional cuyo objetivo principal es mejorar las condiciones de vivienda de los "sin casa". La constitución de un comité de vivienda otorga a los vecinos reconocimiento institucional. Una vez que se finalice la urbanización, los comités deberán conformar otra organización, la junta de vecinos, que es una organización territorial.
} 
las organizaciones comunitarias de Manuel Bustos se organizó previamente a la toma de terrenos. El resto de los comités se conformaron una vez que las familias ya residían en el asentamiento.

Al finalizar los años 90, el campamento Manuel Bustos ya era un asentamiento compuesto por varias familias cuyos orígenes son múltiples: algunas familias son migrantes nacionales, especialmente de Santiago, aunque en su mayoría provienen de otras zonas de Viña del Mar. Sus pobladores llegaron a vivir al campamento como una alternativa a su condición de allegados, porque no cumplían los múltiples requisitos para acceder a la vivienda social, o porque el alto costo de los arriendos y los bajos sueldos no les permiten el desarrollo de una vida digna (Angelcos \& Pérez, 2017). También forman parte de Manuel Bustos algunas familias que fueron beneficiarias de vivienda social, pero las malas condiciones del barrio, la delincuencia y el hacinamiento les han hecho abandonarla y 'regresar' al campamento (Morales et al., 2017). Estas situaciones, tan disimiles, componen una heterogénea población en Manuel Bustos.

Si consideramos que los habitantes de Manuel Bustos carecen del abastecimiento de agua potable, alcantarillado, iluminación pública, pavimentación y se mantienen en una situación de tenencia irregular de la tierra, su población cumple todos los requisitos para estar dentro del sector más pobre de la población, a pesar de estas condiciones, difícilmente sus residentes podrían ser definidos como outsiders. Lejos de ser marginales, los habitantes de los campamentos experimentan distintos tipos de vulnerabilidad expresadas en trayectorias ascendentes y descendentes (Imilán et al., 2020). Es más, algunos estudios señalan que, en los países del sur global, la residencia en estos territorios contribuye a la movilidad social (Caldeira, 2017), pues eximirse del pago de arriendo o de servicios constituye un aporte significativo a familias altamente vulnerables, que pueden destinar dichos fondos a alimentación, transporte o escolaridad.

A pesar de las adversidades del territorio, caracterizado por ser un terreno agreste, empinado y lleno de quebradas, las viviendas en Manuel Bustos comienzan a masificarse, alcanzando su mayor flujo migratorio para los años 2000. Los primeros comités que se formaron en el territorio fueron organizando la llegada de los nuevos requirentes de terreno. Algunos comités solicitaron, incluso, referencias y certificado de antecedentes para aceptar a las nuevas familias. La organización comunitaria fue trazando las calles y definiendo los lotes, siendo en práctica agentes de urbanización a través de la construcción de sus viviendas, sus barrios, su ciudad (Caldeira, 2017).

La organización comunitaria y la autogestión han sido los pilares para resolver necesidades fundamentales. Si bien en el periodo de instalación del campamento se ejerce una mezcla entre práctica individual (en la decisión y gestión de la toma del terreno y en la construcción de la vivienda), y práctica colectiva (porque los vecinos se ayudan en la construcción); una vez instalados los vecinos en el terreno, las prácticas colectivas toman fuerza para resolver, por ejemplo, el abastecimiento de servicios básicos, pues parece ser que, como en muchas ciudades latinoamericanas, para los sectores más pobres dicha provisión queda en las manos de la comunidad local (Besana et al., 2015:81). Es así que se activan los saberes entre los vecinos: quién más sabe de construcción traspasa sus conocimientos a familiares y vecinos (Castillo, 2014), con el fin de resolver los problemas de la comunidad. Estas prácticas colaborativas contribuyeron a la construcción de una especie de acera o escalera que facilita el tránsito por las calles de tierra, fundamental para la movilidad durante el invierno. 
Lo más difícil para sus habitantes ha sido la provisión del agua, pues, en sus primeros años, debían bajar el cerro con baldes a buscar agua a las vertientes naturales que se encontraban en la parte baja. Con el tiempo, la mayor parte del campamento se abasteció de agua potable, pues, entre todos los vecinos rompieron parte de la matriz de agua potable que abastecía a otros sectores de Viña del Mar, e instalaron tubos para tener agua domiciliaria, conectándose a la matriz de la empresa de aguas. Sin embargo, en algunos sectores más altos del cerro, el agua no alcanza a llegar y aún hoy dependen de la provisión de agua potable suministrada por camiones cisterna.

Durante los primeros años de instalación en el terreno, sus residentes debieron enfrentar episodios de represión policial y erradicación, incluso a veces, vieron destruido lo recientemente construido. En una forma de transitoriedad permanente (Rolnik, 2017), los residentes se acostumbraron a este por mientras, el cual poco a poco se va volviendo permanente, la represión cesa y las viviendas se hacen más estables. Manuel Bustos ha estado constantemente en construcción; las viviendas, las calles, el barrio nunca son finitos, siempre hay eventuales mejoras. La vivienda se convierte en el destino de las escasas inversiones familiares que, en las lógicas de la autoconstrucción, implica la adquisición de materiales. En la urbanización periférica, el consumo y el crédito son claves, por lo que las instituciones bancarias o casas comerciales ${ }^{9}$ están prontas a financiar estas compras usualmente con altas tasas de interés (Caldeira, 2017:9). De hecho, el endeudamiento en Manuel Bustos es alto, entre otras cosas, debido a la construcción o mejoramiento de sus viviendas.

La comunidad de Manuel Bustos ha construido no sólo su propia ciudad 'tabla por tabla', sino que también se han organizado socialmente para disputar la vivienda, la propiedad, infraestructura y servicios: para disputar, en otras palabras, el derecho a la ciudad (Caldeira \& Holston, 2015:2002). Para ello, la asociatividad y a la organización comunitaria, expresados en los comités de vivienda, han sido fundamentales para construir la infraestructura del campamento. Con todo, se debe notar que, inicialmente, cada uno de los comités de vivienda trabajaba autónomamente, resolviendo separadamente los problemas que se les presentaban. De hecho, los vecinos ni siquiera se reconocían a sí mismos como habitantes de un solo campamento, sino que entendían que su unidad de reconocimiento no era otra que el comité de vivienda. Ciertos comités habían construido una sede social, una especie de vivienda colectiva en la que se desarrollan tanto las reuniones del comité, como la celebración de fiestas y eventos sociales. Sin embargo, durante los primeros años de formación del campamento, cada comité trabajaba autónomamente, sin comunicarse con los comités colindantes.

Se había ocupado el terreno y se habían conformado pequeñas agrupaciones que lograban resolver problemáticas vinculadas a la infraestructura y la vida cotidiana, pero no se veían a sí mismos como una misma comunidad, afligidos por las mismas carencias. Cada dirigente velaba por los intereses de su propio comité. El paso siguiente, entonces, fue construir una comunidad ampliada que agrupase a todos los residentes de este territorio en una misma unidad, esfuerzo que se concreta el año 2008, -con el apoyo de la ONG Techo-, con la conformación de una mesa conjunta en la que participaron los 23 comités de vivienda del campamento.

Ahí recién empezamos a trabajar con otros dirigentes [refiriéndose a la conformación de la Agrupación] y se formó lo que es la mesa conjunta, con presencia de las autoridades 
que les correspondía, Municipalidad, SERVIU, Techo y los dirigentes (Antonia, dirigenta social, 2018).

Esta nueva organización estratégica, representada por su vocería, ha sido la encargada de realizar las gestiones políticas y de negociar con las autoridades. De hecho, su conformación como Agrupación de Comités de Vivienda del Campamento Manuel Bustos ha sido fundamental para lograr posicionarse como un actor relevante ante las instituciones públicas e incluso posicionar la problemática de los campamentos en la agenda pública:

Los dirigentes de Manuel Bustos han contribuido mucho a la estabilidad de la política pública de campamento, porque siempre está en la agenda, ellos siempre están "oye, no se les olvide", "oye, hay que avanzar" (Teresa, representante instituciones públicas, 2018).

Empezamos a agarrar fuerza nosotros los dirigentes, empezamos a hacernos conocidos, empezamos a empoderarnos de nuestro título de dirigente, empezamos a tener mayor capacidad de poder hablar y todo, y saber cómo hacernos escuchar (Margarita, dirigenta social, 2018).

Las autoridades político-administrativas reconocen en la dirigencia social de Manuel Bustos una característica de empoderamiento y alta capacidad de diálogo que les permite moverse en distintos escenarios políticos. En esta capacidad de hacerse escuchar ante las autoridades políticas han sido fundamentales las dirigencias sociales que se han forjado en el campamento, que han logrado romper la hegemonía dominador-dominado (Boldrini \& Gómez, 2014) y transformaron su reclamo en una demanda clara para las autoridades.

\section{Dirigencia social, pilar de la planificación participativa}

Durante toda la historia del campamento, el accionar político en el territorio no ha estado ausente de prácticas clientelares (Auyero, 2001), que intentan promover la instalación de una relación jerárquica y vertical entre los actores sociales (Rehren, 2000). A través de estas prácticas, se ha buscado crear un vínculo de dependencia entre las instituciones, sus políticos y la comunidad. En algunas ocasiones estas prácticas clientelares se han ejercido en Manuel Bustos, por ejemplo, con la entrega de materiales escolares a los comités políticamente más afines. De hecho, estas prácticas clientelares o de cooptación se ejercen en un marco de profundas desigualdades y escases de recursos (Sarmiento et al., 2007). Sin embargo, estas prácticas no se han difundido ampliamente por el territorio y, paralelamente, las dirigencias han logrado constituirse como actores políticos relevantes, reconocidos por las distintas instituciones públicas:

Yo creo que la Manuel Bustos es un ejemplo de una buena organización territorial, una buena organización comunitaria [...] lograron entablar un diálogo directo con el Gobierno Regional, con los municipios, con organizaciones privadas (José, representante instituciones públicas, 2017).

Las dirigencias, en cuanto intermediarias, han logrado traducir las demandas de la comunidad en políticas públicas. Para poder negociar y posicionar los requerimientos comunitarios, 
las dirigencias han debido capacitarse continuamente sobre los aspectos técnicos. Están muy preparadas y conocen en detalle los pasos de la urbanización, la estructura del campamento y las etapas técnicas que seguirán a este proceso. Es más, en su lenguaje, han incorporado conceptos técnicos, como loteo y deslinde, para explicarle a su comunidad de qué modo se ejecutarán los trabajos de urbanización. Las mujeres 'dirigentas' - pues son en su mayoría mujeres- se han formado como verdaderas gestoras vecinales, con competencias para negociar y discutir sobre los instrumentos de planificación y gestión (Castillo, 2014). Conocen a la perfección las herramientas institucionales y los términos técnicos que les competen para la urbanización. Esta formación les permite poder dialogar y levantar sus demandas específicas logrando -a través de su participación activa- transformar e incidir en el accionar de las políticas públicas.

Más aún, algunas dirigencias se esfuerzan por establecer vínculos con los partidos y con las élites políticas para facilitar sus reclamos (Auerbach, 2017). Estos actos de intermediación con políticos y partidos son considerados, por las dirigentes, como acciones sin fines políticos que les permiten cumplir con su labor social; en su discurso, atribuyen esta clase de vínculos a la búsqueda del bien común (Arraigada, 2009), son alianzas instrumentales (Appadurai, 2013). Este tipo de vínculos favorece la inclusión en los espacios de participación política local:

Ellos, por ejemplo, siempre participan en el CORE, el Consejo Regional, del GORE, que es donde están los recursos del FNDR ${ }^{10}$, etcétera. Ellos son activos participantes, van a las sesiones, y tienen su relación con los consejeros y todo, son muy movidos, con los senadores, con diputados, con todo. Tienen los números de teléfono, tienen un tremendo liderazgo. Si son tremendos. Son súper movidos ${ }^{11}$ (Teresa, representante de institución pública, 2017).

Es más, en esta figura, las dirigencias sociales altamente preparadas y con vínculos políticos se convierten en las mediadoras más capacitadas entre las instituciones públicas y la comunidad. Sin embargo, es de notar que esta posición de intermediarias es una relación bidireccional, pues las dirigencias son, por un lado, las encargadas de hacer llegar las demandas de la comunidad a las instituciones, pero, por el otro lado, también de interpretar las acciones del Estado en términos comprensibles para la comunidad:

Los dirigentes son los principales comunicadores del trabajo que se está haciendo, tranquilizadores también cuando se requiere, nexo con nosotros (Carlos, representante instituciones, 2017).

Convertidas en las intermediarias de las instituciones públicas, estas dirigentes sociales llegan a ser instrumentos de gestión y ejecución de la política pública, piezas clave para su buen funcionamiento. En muchos casos, las dirigencias asumen tareas y responsabilidades públicas para resguardar la ejecución de la política. En primer lugar, son las encargadas de la comunicación y difusión de las acciones del Estado en el proceso de urbanización. En segundo lugar, tienen el poder de validar ante el Estado la antigüedad de las familias; por añadidura, cumplen también

El Fondo Nacional de Desarrollo Regional es un programa de inversiones públicas destinado a financiar acciones en distintos ámbitos de infraestructura social y económica de la región, con el objetivo de obtener un desarrollo territorial armónico y equitativo.

La voz movidos, en este contexto, alude a la capacidad de gestión de los dirigentes. 
la tarea de informantes. En la práctica, el Estado empodera a las dirigencias de cada uno de los comités para identificar a las familias que han llegado de manera reciente al campamento. Las dirigentes deben informarle al Estado cuáles son las familias que, aunque estén fuera del catastro del año 2011, actualizado el 2016, son residentes antiguos; aunque, también, deben informar de aquellas familias que están fuera del catastro porque, efectivamente, han llegado recientemente al campamento, pues inicialmente solo las familias antiguas ${ }^{12}$ serían beneficiadas con la urbanización. Es como si la paciencia, la espera (Appadurai, 2013), el esfuerzo y el sacrificio (Pérez, 2017) fuesen requisitos para merecer habitar en la ciudad formal.

El poder que se les otorga a las dirigentes les permite también tener una herramienta para enfrentar una de sus principales preocupaciones, el narcotráfico. Todas ellas quieren evitar que en la urbanización sean incluidas personas indeseables. Para evitarlo, deben notificar a las instituciones públicas cuál es la familia en cuestión, iniciándose, con ello, un proceso de persecución policial y posterior desalojo. Así, el Estado delega en las dirigentes la función de fiscalizar estos delitos y ellas asumen una especie de responsabilidad higienista en su comunidad, como si fuesen las encargadas de limpiar el barrio de la droga. Por estas razones, las familias que son desalojadas responsabilizan a las dirigentes de sus comités por el desalojo, quienes han recibido amenazas:

En mi comité hay familias que tienen que desalojar y a mí me tienen amenazada, ime tiran piedras! Yo fui atacada por una familia que desalojaron (Nota de campo, mesa ampliada, 16 de agosto de 2017).

Me amenazó, me dijo: "mis amigas ahora están en la cárcel, pero cuando salgan, te van a venir a ver". Yo al otro día fui a hacer una denuncia, pero no en contra de la cabra, ${ }^{13}$ sino en contra del SERVIU, porque son ellos los que nos tienen que proteger a nosotras las dirigentas [sic], nosotras no podemos sufrir agresiones por lo que estamos haciendo. iEl SERVIU nos tiene que proteger! -comenta Ana. (Nota de campo, reunión de comité, 18 de abril de 2017).

Se denota así una clara transferencia de responsabilidades desde el Estado hacia las dirigencias sociales, quienes son las responsables últimas por velar que el proceso de urbanización ocurra en modo ordenado. El poder entregado por las instituciones se transforma en una trampa higienista en la que terminan involucradas tanto las familias indeseables como las propias dirigencias encargadas de ejercer las tareas de limpieza.

Por otro lado, una de las más significativas disputas que las organizaciones de Manuel Bustos han tenido que dar, ha sido el hacer entender a la institucionalidad, que sus demandas no se vinculaban -sólo- a la propiedad de la vivienda. De hecho, una vez iniciadas las conversaciones entre la comunidad y el Estado, este último dio como primera respuesta, la erradicación del campamento a través del otorgamiento de subsidios habitacionales, que parecía ser la única salida para

\footnotetext{
La antigüedad de una familia la determina, inicialmente, el catastro realizado el año 2011. Sin embargo, después de considerables presiones por parte de algunas dirigentes se logró relativizar el catastro, con la declaración de las dirigentes y otros documentos que certifiquen su antigüedad. Asi también, con la firma del Protocolo de Urbanización el año 2017 se logró considerar también a las familias que forman parte de la actualización del catastro realizada el 2016.

13 Cabra: palabra utilizada para referirse a una mujer, joven o niña. En este caso, es usado para aludir a la mujer que amenazó a la dirigente.
} 
acceder a la vivienda. Sin embargo, las dirigencias fueron enfáticas en sus demandas: pretendían la regularización de la tenencia y la instalación de infraestructura básica. Sabían que estaban en terrenos públicos, por lo que la radicación era posible.

Finalmente, en abril de 2017 se firma del Protocolo de Urbanización, el cual contempla un Plan de Obras de Urbanización y un Plan de Regularización de la Propiedad, incluyendo la sesión de terrenos a las familias del campamento. Las dirigencias han estado activas, y participando de todo el proceso, insistiendo en que la urbanización debía ser lo menos invasiva posible, respetando la estructura y la distribución de las viviendas. Cada paso de la urbanización implica una serie de reuniones en los mismos comités, donde se acordó, por ejemplo, mantener la infraestructura de las viviendas, puesto que contienen las inversiones familiares, además de las memorias y los lazos de solidaridad generados en su construcción; aspectos que la construcción de una vivienda nueva no compensa.

La ejecución del proceso de urbanización no ha estado exenta de tensiones, entre las cuales se destaca la elaboración del plano de loteo, el atraso de los plazos para concretar las obras de urbanización y las decisiones respecto a, por ejemplo, quiénes pueden o no quedarse en el territorio (Sepúlveda, 2019). De hecho, entre 2017 e inicios del 2020, Manuel Bustos ha visto lentos avances en torno a la urbanización: se aprobó el plano de loteo, los pobladores han adaptado su terreno a este plano, se ha iniciado la cesión terrenos a los pobladores y se han comenzado algunas labores de infraestructura. Estos lentos avances ponen prueba la paciencia y la espera de los pobladores, convirtiéndose en una nueva forma de violencia institucional.

\section{Reflexiones finales}

El fin de los campamentos en Chile fue una quimera propia del modelo de desarrollo chileno de los años 80 y 90, que en la actualidad hace patente sus falencias. Durante décadas, las instituciones públicas en Chile negaron el crecimiento de estos territorios. Sin embargo, los campamentos, silenciosos e invisibles, permanecieron y se radicaron en los terrenos, fuera de la ley y de la atención pública.

La política pública chilena considera, tardíamente, la urbanización de asentamientos informales. Sin duda, el proceso que está atravesando Manuel Bustos es innovador en Chile, pues logra romper con la lógica top-down de la mayor parte de las políticas de vivienda, considera a la comunidad - y no a la familia - como unidad de intervención, permite el reconocimiento de las memorias y vínculos con territorio habitado e incluye participativamente a la comunidad en los procesos de planificación y control de la ejecución.

Sin embargo, es necesario tener presente que la participación de la comunidad no ha sido una concesión del Estado, sino que más bien responde a una conquista por parte de los movimientos sociales. La urbanización del campamento Manuel Bustos se concreta luego de años de empoderamiento y resistencia. Asimismo, la participación ha sido efectiva porque han sido las dirigencias las que han estado permanentemente exigiendo ser incluidas.

El campo en disputa en la urbanización del campamento Manuel Bustos no se refiere, entonces, al derecho a la vivienda o al de tenencia, sino que es una lucha más compleja frente al Estado 
y sus instituciones. Las dirigencias de Manuel Bustos lograron disputar el derecho a una vivienda digna, en el territorio de sus propias memorias y lazos comunitarios. Más aún, lograron hacerlo en una comuna en donde el precio del suelo urbanizado es de alto de valor. Así, lograron transformar sus luchas por la vivienda a una lucha por el derecho a la ciudad.

Ahora bien, el gran desafío de poner en marcha un modelo de política pública participativa requiere transformar las prácticas que el Estado ha tenido durante los últimos años, caracterizado por la invisibilización de las organizaciones sociales al momento de construir ciudad. Las tensiones que han surgido a lo largo del camino, han provocado que las dirigencias estén atentas a las labores realizadas y en diálogo permanente con las instituciones.

A nuestro parecer, esta hábil gestión de la participación comunitaria se sustenta en una sólida trayectoria de producción social del hábitat, que incluye prácticas colaborativas arraigadas, de una elaborada comprensión del propio territorio, pero también a la formación de dirigencias empoderadas, con capacidad de diálogo en distintos frentes (la comunidad, el gobierno local, las instituciones gubernamentales). Asimismo, ha sido fundamental la apertura de las instituciones hacia la transformación de la política pública de asentamientos informales. La conjugación de estos factores ha logrado llevar a cabo una política pública participativa como pocas.

Frente a la inminente finalización de la urbanización, el mayor desafío de la organización comunitaria en Manuel Bustos es lograr encontrar aspectos que permitan mantener la unión entre vecinos, encontrando nuevos espacios para comprometerse por una necesidad común, que trascienda la urbanización.

Por otro lado, en cuanto a las acciones del Estado, si bien es notable el proceso de urbanización en Manuel Bustos, este ha sido tratado como un caso excepcional de urbanización de asentamientos informales; la tendencia sigue siendo la erradicación. Si bien el caso no es la panacea, especialmente por los retrasos y largos tiempos que ha implicado, esta experiencia evidencia lo valioso de planificar la ciudad con sus propios residentes, especialmente cuando han sido ellos quienes, desde la insurgencia, han construido sus propias ciudades.

\section{Referencias bibliográficas}

AUYERO, J. La política de los pobres. Las prácticas clientelistas del peronismo. Buenos Aires, Argentina: Editorial Manantial, 2001.

AUYERO, J. \& SWISTUN, D. Inflamable. Estudio del sufrimiento ambiental. Buenos Aires, Argentina: Editorial Paidós, Tramas Sociales 45, 2008.

ARRAIGADA, E. "El sustento relacional de los liderazgos locales". Profesor guía: Emmanuelle Barozet. Tesis de Magister. Universidad de Chile, Facultad de Ciencias Sociales, Santiago, 2009.

ARQUEROS MEJICA, M., GIL Y DE ANSO, M., MENDOZA, M. \& ZAPATA, M. Córdoba y Mendoza: dos casos para pensar la producción social del hábitat. Revista INVI, 2009, N62, p. 21-48. 
APPADURAI, A. El futuro como hecho cultural. Ensayos sobre la condición global. Buenos Aires, Argentina: Editorial Fondo de Cultura Económica, 2013.

ANGELCOS, N. \& PÉREZ, M. De la desaparición a la reemergencia: Continuidades y rupturas del movimiento de pobladores en Chile. Latin American Research Review. 2017, No1, p. 94-109.

AUERBACH, A. Neighborhood Associations and the Urban Poor: India's Slum Development Committees. World Development, 2017, Vol.96, p. 119-135.

BRAIN, I.; PRIETO, J. \& SABATINI, F. Vivir en Campamentos: ¿Camino hacia la vivienda formal o estrategia de localización para enfrentar la vulnerabilidad? Revista EURE, 2010, №109, p. 111-114.

BOLDRINI, P. \& GÓMEZ, C. Participación popular en la producción del hábitat. El caso del Área Metropolitana de Tucumán-Argentina. Revista de Geografía Norte Grande, 2014, №57, p. 67-82.

BESANA, P.; GUTIÉRREZ, R. \& GRINBERG, S. Pobreza urbana, comunidad local y Estado-socio en Argentina: la provisión de servicios públicos en un asentamiento de la Región Metropolitana de Buenos Aires. Revista Mexicana de Ciencias Políticas y Sociales, 2015, №225, p. 79-102.

CORTÉS, A. El movimiento de pobladores chilenos y la población La Victoria: ejemplaridad, movimientos sociales y el derecho a la ciudad. Revista EURE, 2014, No119, p. 239-260.

CASTILLO, M. Competencias de los pobladores: potencial de innovación para la política habitacional chilena. Revista INVI, 2014, Nº1, p. 79-112.

CALDEIRA, T. \& HOLSTON, J. Participatory urban planning in Brazil. Urban Studies, 2015, N¹1, p. 2001-2017.

CALDEIRA, T. Peripheral urbanization: Autoconstruction, transversal logics, and politics in cities of the global south. Environment and Planning D: Society and Space, 2017, Nº1, p. 3-20.

CONTRERAS, Y,; NEVILLE, L. \& GONZÁLEZ, R. In-formality in access to housing for Latin American migrants: a case study of an intermediate Chilean city. International Journal of Housing Policy, 2019, N³, p. 411-435.

DUCCl, M. Chile: El lado oscuro de una política de vivienda exitosa. Revista EURE, 1997, №69, p. 99-115

DI VIRGILIO, M. \& RODRÍGUEZ, M. Producción social del hábitat: Abordajes conceptuales, prácticas de investigación y experiencias en las principales ciudades del Cono Sur. Buenos Aires, Argentina: Editorial Café de las Ciudades, 2013.

GILBERT, A. Helping the poor through housing subsidies: Lessons from Chile, Colombia and South Africa. Habitat International, 2004, Nº1, p. 13-40. 
GONZÁLEZ, G. El problema de la vivienda y la Producción Social del Hábitat del Sur al Norte de América Latina. En: ARÉBALO, M.; BAZOBERRY, G.; BLANCO, C.; DÍAZ, S.; FERNÁNDE, R.; FLORIAN, A.; GARCÍA, R.; GONZÁLEZ, G.; LANDAETA, G.; MANRIQUE, D.; MIYASHIRO, J.; NOAHOUM, B.; OLSSON, J.; ORTIZ, E.; PESSINA, L.; SUGRANYES, A. \& VILA, C. El camino posible: Producción Social del Hábitat en América Latina. Montevideo: Ediciones Trilce, 2012, p. 239-251.

GARCÉS, M. El movimiento de pobladores durante la Unidad Popular, 1970-1973. Atenea, 2015, N512, p. 33-47.

IMILÁN, C.; OSTERLING, E.; MANSILLA, P. \& JIRÓN, P. El campamento en relación con la ciudad: informalidad y movilidades residenciales de habitantes de Alto Hospicio. Revista INVI, 2020, n³5, p. 57-80.

KOPPELMAN, C. Deeping Demobilization. The State's Transformation of Civil Society in the Poblaciones of Santiago, Chile. Latin American Perspectives, 2016, N 3, p. 46-63.

LANGE, C. El hábitat residencial en perspectiva colaborativa: desafíos para la producción social del conocimiento. En: IMILAN, W.; LARENAS, J.; CARRASCO, G. \& RIVERA, S. (Eds.). ¿Hacia dónde va la vivienda en Chile? Nuevos desafíos en hábitat residencial. Santiago de Chile: FAU, Universidad de Chile y Adrede Editora, 2017, p. 91-104.

LOPEZ-MORALES, E.; FLORES, P. \& OROZCO, H. Inmigrantes en campamentos en Chile: ¿mecanismo de integración o efecto de exclusión? Revista INVI, 2018, Nº94, p. 161-187.

MORALES, E.; BESOAIN, C.; SOTO, A.; PINTO, L.; HIDALGO, K.; FERNÁNDEZ, I., \& BERNAL, V. Retorno al campamento: Resistencia y melancolía en los márgenes de la ciudad formal. Revista INVI, 2017, Nº9, p. 51-75.

MINISTERIO DE VIVIENDA Y URBANISMO, MINVU. "Catastro nacional de campamentos" [en línea]. 2019 [consulta: 27-02-2020]. Disponible en: https://www.minvu.cl/catastro-de-campamentos/

ORTIZ, E. Producción social de vivienda y hábitat: bases conceptuales para una política pública. En: ARÉBALO, M.; BAZOBERRY, G.; BLANCO, C.; DÍAZ, S.; FERNÁNDE, R.; FLORIAN, A.; GARCÍA, R.; GONZÁLEZ, G.; LANDAETA, G.; MANRIQUE, D.; MIYASHIRO, J.; NOAHOUM, B.; OLSSON, J.; ORTIZ, E.; PESSINA, L.; SUGRANYES, A. \& VILA, C. El camino posible: Producción Social del Hábitat en América Latina. Montevideo: Ediciones Trilce, 2012, p. 13-44.

ÖZLER, Ş I. The Concertación and homelessness in Chile: Market-based housing policies and limited popular participation. Latin American Perspectives, 2012, No 4, p. 53-70.

PÉREZ, M. "A New Poblador Is Being Born": Housing Struggles in a Gentrified Area of Santiago. Latin American Perspectives, 2017, No 3, p. 28-45.

REHREN, A. "Clientelismo político, corrupción y reforma del Estado en Chile" [en línea]. Junio 2000 [consulta: 26-02-2020]. Disponible en: https://www.cepchile.cl/cep/site/artic/20160304/ asocfile/20160304093611/refor2_02_rehren.pdf 
RODRÍGUEZ, A. \& SUGRANYES, A. El problema de vivienda de los "con techo". Revista EURE, 2004, N॰91, p. 53-65.

ROLNIK, R. La guerra de los lugares. La colonización de la tierra y la vivienda en la era de las finanzas. Santiago de Chile: Editorial LOM Ediciones, 2017.

SKEWES, J. C. De invasor a deudor: el éxodo desde los campamentos a las viviendas sociales en Chile. En: RODRÍGUEZ, A. \& SUGRANYES, A. Los con techo. Un desafío para la política de vivienda social. Santiago de Chile: Ediciones SUR, 2005, p. 101-122

SARMIENTO, J.; TELLO, C. y SEGURA, R. Ciudadanía, sociedad civil y participación en políticas públicas. La experiencia de los Foros Vecinales de Seguridad en el municipio de La Plata. Revista Katal. Florianópolis, 2007, №2, p.187-196.

SALCEDO, R. The Last Slum: Moving from Illegal Settlements to Subsidized Home Ownership in Chile. Urban Affairs Review, 2010, N¹, p. 90-118.

SUGRANYES, A. La reconstrucción social del subsidio habitacional. En: ARÉBALO, M.; BAZOBERRY, G.; BLANCO, C.; DÍAZ, S.; FERNÁNDE, R.; FLORIAN, A.; GARCÍA, R.; GONZÁLEZ, G.; LANDAETA, G.; MANRIQUE, D.; MIYASHIRO, J.; NOAHOUM, B.; OLSSON, J.; ORTIZ, E.; PESSINA, L.; SUGRANYES, A. \& VILA, C. El camino posible: Producción Social del Hábitat en América Latina. Montevideo: Ediciones Trilce, 2012, p. 45-58.

SEPÚLVEDA, K. Experiencias de urbanización: El caso del campamento Manuel Bustos (Viña del Mar, Chile). Revista Faro, 2019, N³0, p. 6-19.

ZENTENO, T. Políticas de contraste a la marginalidad urbana. El caso de Santiago de Chile y São Paulo. En: SEHTMAN, A. Y ZENTENO, E. (Eds.). Continuidades, rupturas y emergencias. Las desigualdades urbanas en América Latina. Ciudad de México: Universidad Nacional Autónoma de México, 2015, p.159-174. 